\title{
Blocking hexose entry into glycolysis activates alternative metabolic conversion of these sugars and upregulates pentose metabolism in Aspergillus nidulans
}

Claire Khosravi', Evy Battaglia', Roland S. Kun', Sacha Dalhuijsen², Jaap Visser ${ }^{1,4}$, María Victoria Aguilar-Pontes ${ }^{1}$, Miaomiao Zhou', Heino M. Heyman³, Young-Mo Kim ${ }^{3}$, Scott E. Baker ${ }^{3}$ and Ronald P. de Vries ${ }^{1,2^{*}}$ (D)

\begin{abstract}
Background: Plant biomass is the most abundant carbon source for many fungal species. In the biobased industry fungi, are used to produce lignocellulolytic enzymes to degrade agricultural waste biomass. Here we evaluated if it would be possible to create an Aspergillus nidulans strain that releases, but does not metabolize hexoses from plant biomass. For this purpose, metabolic mutants were generated that were impaired in glycolysis, by using hexokinase ( $h \times k A)$ and glucokinase $(g / k A)$ negative strains. To prevent repression of enzyme production due to the hexose accumulation, strains were generated that combined these mutations with a deletion in creA, the repressor involved in regulating preferential use of different carbon catabolic pathways.

Results: Phenotypic analysis revealed reduced growth for the $h \times k A 1$ glkA4 mutant on wheat bran. However, hexoses did not accumulate during growth of the mutants on wheat bran, suggesting that glucose metabolism is re-routed towards alternative carbon catabolic pathways. The creA $\Delta 4$ mutation in combination with preventing initial phosphorylation in glycolysis resulted in better growth than the $\mathrm{x} \times \mathrm{kA} \mathrm{g} / \mathrm{kA}$ mutant and an increased expression of pentose catabolic and pentose phosphate pathway genes. This indicates that the reduced ability to use hexoses as carbon sources created a shift towards the pentose fraction of wheat bran as a major carbon source to support growth.
\end{abstract}

Conclusion: Blocking the direct entry of hexoses to glycolysis activates alternative metabolic conversion of these sugars in $\mathrm{A}$. nidulans during growth on plant biomass, but also upregulates conversion of other sugars, such as pentoses.

Keywords: Central carbon catabolism, Aspergillus nidulans, Glycolysis, Pentose catabolic pathway, Plant biomass degradation

\section{Background}

Plant biomass is the main renewable material on earth and the major starting material for several industrial processes. In nature, Aspergillus degrades plant biomass polysaccharides to obtain monomeric sugars that can serve as a carbon source. Aspergillus is able to secrete enzymes that can hydrolyse polysaccharides into pentoses and hexoses [1], which can be taken up by the fungus. Aspergillus then uses

\footnotetext{
* Correspondence: r.devries@westerdijkinstitute.nl

${ }^{1}$ Fungal Physiology, Westerdijk Fungal Biodiversity Institute \& Fungal Molecular Physiology, Utrecht University, Uppsalalaan 8, 3584, CT, Utrecht, The Netherlands

${ }^{2}$ Microbiology, Utrecht University, Padualaan 8, 3584, CH, Utrecht, The Netherlands

Full list of author information is available at the end of the article
}

a variety of catabolic pathways to efficiently convert all monomeric components of plant biomass. D-glucose is a hexose that can be phosphorylated to glucose-6-phosphate by either glucokinase (GlkA) [2] or hexokinase (HxkA) [3] in Aspergillus nidulans. Glucose-6-phosphate can either enter the pentose phosphate pathway (PPP) or be converted to fructose-6-phosphate by phosphoglucose isomerase (SwoM) [4] and enter glycolysis. D-fructose is phosphorylated by hexokinase (HxkA) to fructose-6-phosphate, which can also enter glycolysis $[3,5]$.

The critical step in bio-ethanol production is the release of sugars from plant material, because the enzymes used in this process are expensive to produce and purify. A possibility to optimize the process is to create a fungal strain 
that can hydrolyse the polymeric fraction but cannot utilize fermentable sugars such as D-glucose and Dfructose. With such a strain the plant degrading enzymes do not have to be produced and purified in a separate environment, but the fungus itself can be used to generate these sugars during growth on plant biomass. Such a 'onepot' bioethanol process has been reported by combining cellulase production and ethanol production using a cocultivation of Acremonium cellulolyticus and Saccharomyces cerevisiae. [6]. Another study in which S. cerevisiae was co-cultured with other fungi also revealed an increase in ethanol production and a reduction of the fermentation time and cost [7].

The genomes of most filamentous fungi contain all the necessary glycolytic genes for conversion of D-glucose and D-fructose. The aim of this study was to combine a carbon catabolite derepressed strain with a glucose and fructose non-consuming strain, redirecting metabolism towards consumption of other sugars (e.g. pentoses), and analyze the effect of these strains on a complex substrate. Previous phenotypic analysis of glucokinase and hexokinase mutants from Aspergillus nidulans demonstrated that at least one of them is required for normal growth on D-fructose or D-glucose. [3, 5]. It has also been shown that growth of an A. fumigatus $\triangle h x k A \Delta g l k A$ strain is reduced on D-glucose and D-fructose [8] and that the $A$. nidulans hxkA1 glkA1 mutant is unable to phosphorylate D-glucose and D-fructose [5]. Phosphorylation of hexose sugars by both glucokinase and hexokinase was shown to play a role in mediating carbon catabolite repression (CCR) via CreA, but it was not essential [5].

In order to prevent hexose consumption we blocked entry to glycolysis of these sugars by using a hexokinase/ glucokinase mutant. As accumulation of hexoses could activate carbon catabolite repression of genes encoding plant biomass degrading enzymes, we combined these mutations with a nul-mutation in creA caused by a partial deletion that was previously described [9]. The resulting mutant strains have been analysed on wheat bran with regard to growth, extra - and intracellular sugar accumulation, extracellular enzyme production, and gene expression.

\section{Methods}

\section{Chemicals}

All chemicals and reagents were acquired from SigmaAldrich unless otherwise noted.

\section{Strains, media and growth conditions}

All $A$. nidulans strains used in this study are listed in Table 1 and were grown in minimal medium (MM) or complete medium (CM) with addition of a carbon source [10]. The nitrogen source used in our media is sodium nitrate $(6 \mathrm{~g} / \mathrm{l})$. For all the analyses performed, two biological replicates were used, except for the metabolomics where three biological replicates were used. For the growth on plates, $1.5 \%(w / v)$ agar was added to the medium. For the production of spores, the strains were cultivated on plates containing CM supplemented with $1 \%$ xylose and incubated at $37^{\circ} \mathrm{C}$ in the dark for 5 days. The spores were harvested in ACES buffer and counted with haemocytometer (THOMA) under 40x magnifications. Square plates of MM plus 1\% polysaccharide or $3 \%$ crude carbon source were used for growth profile. When necessary, the medium was supplemented with pyridoxine (pyro, 0.2 g.L $\mathrm{L}^{-1}$ ), pantothenic acid (panto, 0.1 g.L $\mathrm{L}^{-1}$ ) and para-aminobenzoic acid (paba, $0.2 \mathrm{~g}$. $\left.\mathrm{L}^{-1}\right)$. The plates were inoculated with $2 \mu$ spore solutions at $5 \times 10^{5}$ spores $/ \mathrm{ml}$ and incubated at $37{ }^{\circ} \mathrm{C}$ during $3-4$ days. For the transfer experiments, all the strains were pre-grown in $1 \mathrm{~L}$ Erlenmeyer flasks that contained $200 \mathrm{ml}$ of $\mathrm{CM}$ plus $2 \%$ D-xylose. Liquid cultures were inoculated with $10^{6}$ spores $/ \mathrm{ml}$ and shaken at $250 \mathrm{rpm}$ in a rotary shaker. After $16 \mathrm{~h}$, the mycelium was harvested by filtration, washed twice with $\mathrm{MM}$ and transferred to $250 \mathrm{ml}$ Erlenmeyer flasks containing $50 \mathrm{ml} \mathrm{MM}$ plus $1 \%$ wheat bran $(w / v)$ and the appropriate supplements, at $37{ }^{\circ} \mathrm{C}$. The mycelium was harvested by vacuum filtration and culture samples were taken after 2,8 and $24 \mathrm{~h}$ of incubation. The mycelium samples were dried between tissue paper and frozen in liquid nitrogen. For RNA analysis only the $2 \mathrm{~h}$ samples were used. The supernatant was kept at $-20{ }^{\circ} \mathrm{C}$ for sugar and enzymatic analysis.

\section{Sexual crossing of $A$. nidulans}

Spores of the $A$. nidulans strains were harvested in saline tween buffer. Sexual crosses of the strains were performed as described previously [11]. Selection of the progeny was based on the inability to grow on glucose and fructose for the hexokinase glucokinase double mutant and a halo around a colony grown on starch plus D-glucose for the creA mutation. Selection of the triple mutant was based on the inability to grow on starch plus D-glucose and the ability to grow on starch plus D-xylose with the formation of a halo around the colony.

\section{Enzyme and reducing sugar assays}

The concentration of the sugars in the media was measured by a reducing sugar assay as previously described [12] with modifications: samples were mixed with an equal volume of 3,5-dinitrosalicylic acid (DNS), incubated at $95^{\circ} \mathrm{C}$ for $30 \mathrm{~min}$ and absorbance were measured at $540 \mathrm{~nm}$ using a microtiter plate reader (FLUOstar OPTIMA, BGM Labtech).

Extracellular enzyme activity was measured in a total volume of $100 \mu \mathrm{l}$ using $0.01 \% p$-nitrophenol (PNP) linked substrates, $20 \mu \mathrm{l}$ of the culture samples and $50 \mu \mathrm{l}$ of $50 \mathrm{mM}$ sodium acetate pH 5.0. Samples were incubated in microtiter plates for $1 \mathrm{~h}$ at $37^{\circ} \mathrm{C}$. Reactions were stopped by addition of $100 \mu \mathrm{l} 0.25 \mathrm{M} \mathrm{Na}_{2} \mathrm{CO}_{3}$. Absorbance was measured at $405 \mathrm{~nm}$ in a microtiter plate reader (FLUOstar OPTIMA, BMG Labtech). The extracellular enzyme 
Table 1 Aspergillus nidulans strains used in this study. (In bold are the strains that were used in all experiments)

\begin{tabular}{|c|c|c|c|}
\hline Name & CBS accession number & Genotype & References \\
\hline UUAM101.08 & CBS 141343 & yA2 & This study \\
\hline UUAM104.47 & CBS 141344 & creA $\triangle 4$, pyroA4 & This study \\
\hline UUAM102.37 & CBS 141756 & hxkA1 glkA4, yA2, pabaA1, pyroA4 & This study \\
\hline UUAM104.87 & CBS 141447 & creA $\triangle 4$ hxkA1 glkA4, yA2, pyroA4 & This study \\
\hline UUAM101.24 & & yA2, pyroA4 & This study \\
\hline UUAM102.71 & & hxkA1 glkA4, yA2, pyroA4 & This study \\
\hline NW299 & & glkA4, yA2, pyroA4 & [5] \\
\hline NW193 & & $h \times k A 1$ glkA4, pabaA1 & {$[5]$} \\
\hline V148 & & $\operatorname{cre} A \Delta 4$, pantoA1 & This study \\
\hline
\end{tabular}

activity was calculated using a standard curve ranging from 0 to $40 \mathrm{nmol} p$-nitrophenol per assay per volume. Absorbance measurements for all assays were performed in triplicate. Statistical significance for all enzyme and reducing sugar assays was determined by t-test and the Holm-Sidak method (alpha $=0.05$ ) using GraphPad Prism version 7 for Mac, GraphPad Software, www.graphpad.com.

\section{Monosaccharide analysis}

Monosaccharide analysis was performed for all strains, after $2 \mathrm{~h}, 8 \mathrm{~h}$ and $24 \mathrm{~h}$ of cultures in wheat bran. The culture supernatant was diluted 10-fold in MilliQ water prior to analysis. The monosaccharides were analyzed from peak areas in HPAEC-PAD (Dionex ICS-5000+ system; Thermo Scientific) equipped with CarboPac PA1 column $(2 \times$ $250 \mathrm{~mm}$ with $2 \times 50 \mathrm{~mm}$ guard column; Thermo Scientific). The column was pre-equilibrated with $18 \mathrm{mM} \mathrm{NaOH}$ followed by a multi-step gradient: 0-20 min: $18 \mathrm{mM} \mathrm{NaOH}$, 20-30 min: 0-40 mM NaOH and 0-400 mM sodium acetate, $30-35$ min: $40-100 \mathrm{mM} \mathrm{NaOH}$ and $400 \mathrm{mM}$ to $1 \mathrm{M}$ sodium acetate, $35-40 \mathrm{~min}: 100 \mathrm{mM} \mathrm{NaOH}$ and $1 \mathrm{M}$ to $0 \mathrm{M}$ sodium acetate followed by re-equibration of $18 \mathrm{mM}$ $\mathrm{NaOH}$ for $10 \mathrm{~min}\left(20{ }^{\circ} \mathrm{C}\right.$; flow rate: $\left.0.30 \mathrm{~mL} / \mathrm{min}\right)$. $2.5-$ $200 \mathrm{mM}$ D-glucose, D-fructose, L-arabinose, D-xylose, Dmannose, L-rhamnose, D-galactose, D-glucuronic acid, and D-galacturonic acid (Sigma-Aldrich) were used as standards for quantification [13].

\section{RNA extraction, CDNA library preparation and RNA-seq}

Total RNA was extracted from mycelium ground in a Tissue Lyser (QIAGEN) using TRIzol reagent (Invitrogen) according to the instructions of the manufacturer. RNA integrity and quantity were analyzed both on a $1 \%$ agarose gel using gel electrophoresis and with an Agilent 2100 Bioanalyzer (Agilent Technologies). BGI Tech Solutions Co., Ltd. (Hong Kong) performed the cDNA library preparation and sequencing reactions. Throughout the process, Illumina library preparation, clustering, and sequencing reagents were used following the manufacturer's recommendations (http://illumina.com).
On average 51 bp sequenced single-end reads were obtained, producing approximately $570 \mathrm{MB}$ raw yields for each sample. Principal component analysis (PCA) was performed to show the reproducibility of our samples (Additional file 1: FigureS5).

\section{RNA-seq data analysis and functional annotation}

We produced raw reads from the original image data by base calling. The adaptor sequences, highly ' $\mathrm{N}$ ' containing reads ( $>10 \%$ of unknown bases) and low quality reads (more than $50 \%$ bases with quality value of $<5 \%$ ) were removed after data filtering. After data filtering, in average, $\sim$ 95\% clean reads remained in each sample. Clean reads were then mapped to the genome of $A$. nidulans (AspGD) using SOAPALIGNER/SOAP2 [14]. In the alignment, no more than two mismatches were allowed. On average, $90 \%$ total mapped reads to the genome was achieved. The gene expression level was calculated by using FPKM method [15]. Genes with expression value higher than 150 were considered highly expressed (approximately top 5\%) and differential expression was identified by CyberT bayesian ANOVA algorithm [16] with a cut-off value of fold change $>1.5$ and $P$-value (corrected by multiple tests) $<0.05$. The RNA-seq data have been submitted to Gene Expression Omnibus (GEO) [17] with accession number: GSE94775.

Orthologous genes between Aspergillus niger CBS 513. 88 and Aspergillus nidulans FGSC A4 were obtained from AspGD [18] and FunCat [19] functional annotation was mapped accordingly. Expression higher than 150 RPKM that had a fold change of at least 1.5 between creA $\triangle 4$ $h x k A 1 g l k A 4$ vs $c r e A \triangle 4$ and $h x k A 1 g l k A 4$ vs reference was analyzed regarding the functional annotation.

\section{Quantitative RT-PCR (qRT-PCR) validation}

RNA samples for RNA-seq experiments were used for qRT-PCR. cDNA was prepared from total RNA $(2.5 \mu \mathrm{g})$ using Thermoscript RT (Invitrogen) according to the instructions of the manufacturer. Primer Express 3.0 software (Applied Biosystems) was used to design the sequences of all primers for qRT-PCR analysis, which were tested to 
determine the optimal primer concentrations and efficiency. Combinations of the $50 \mathrm{nM}, 300 \mathrm{nM}$ and $900 \mathrm{nM}$ (final concentration) per primer pair were checked and based on the dissociation curve the optimal primer concentration per primer pair was set. The primer sequences and optimal concentrations of the tested genes and the reference gene are listed in Additional file 2: Table S5. qPCR analysis was performed by using the ABI 7500 fast realtime PCR system (Applied Biosystems). $20 \mu \mathrm{l}$ reactions consisted of $2 \mu \mathrm{l}$ forward and reverse primers at optimal concentration, $20 \mathrm{ng}$ cDNA sample, $10 \mu \mathrm{l}$ ABI Fast SYBR Master Mix (Applied Biosystems), and water to a final volume of $20 \mu \mathrm{l}$. The cycling parameters were $95{ }^{\circ} \mathrm{C}$ for $20 \mathrm{~s}$, followed by 40 cycles of $95{ }^{\circ} \mathrm{C}$ for $3 \mathrm{~s}$ and $60{ }^{\circ} \mathrm{C}$ for $30 \mathrm{~s}$. A dissociation curve was generated to verify that a single product was amplified. Transcript levels were normalized against the glyceraldehyde-3-phosphate dehydrogenase gene (gpdA; AN8041), expression and quantified according to the formula $2-(\mathrm{Ct}$ gene $\mathrm{X}-\mathrm{Ct}$ gpd) [20]. Control reactions included water only and RNA (i.e. not converted to cDNA to detect residual DNA in the sample). Two biological and three technical replicates were analyzed.

\section{Sample preparation for GC-MS metabolomics}

The polar metabolites were extracted using $30 \mathrm{mg}$ biomass from A. nidulans cell pellet, to which $200 \mu \mathrm{L}$ MilliQ water and $800 \mu \mathrm{L}$ of $-20{ }^{\circ} \mathrm{C}$ chloroform: methanol solution (2:1) was added. The cultures were vortexed for $10 \mathrm{~s}$, sonicated for $10 \mathrm{~min}$ and then centrifuged $\left(13,300 \times \mathrm{g}, 4^{\circ}\right.$ C, $5 \mathrm{~min}$ ). After centrifugation $100 \mu \mathrm{L}$ of the top aqueous layer was transferred to glass vials and dried in a vacuum concentrator (CentriVap Concentrator, Labconco). For the analysis of the polar metabolites in the spent media $100 \mu \mathrm{L}$ of spent media was transferred directly to glass vial and dried in a vacuum concentrator.

\section{Chemical derivatization and GC-MS analysis}

Polar metabolites from both the cell pellet and spent media were derivatized as described previously [21]. Briefly, $20 \mu \mathrm{L}$ of methoxyamine hydrochloride in pyridine $(30 \mathrm{mg} / \mathrm{mL})$ was added to each sample, followed by $30 \mathrm{~s}$ of vortexing and $10 \mathrm{~s}$ of sonicating before incubating the samples with shaking (1000 rpm) at $37^{\circ} \mathrm{C}$ for $90 \mathrm{~min}$. Next, $80 \mu \mathrm{L}$ of Nmethyl-N-(trimethylsilyl) trifluoroacetamide (MSTFA) with $1 \%$ trimethylchlorosilane (TMCS) was added to each sample, subsequently, the samples were vortexed for $30 \mathrm{~s}$, sonicated for $10 \mathrm{~s}$ and incubation at $37{ }^{\circ} \mathrm{C}$ with shaking $(1000 \mathrm{rpm})$ for $30 \mathrm{~min}$. Derivatized samples were then transferred to insert before being analyzed by GC-MS in random order. Blanks and FAMEs samples were also included in the analyses for background reference and RT calibration purposes, respectively.

The derivatized metabolites were separated using a HP-5MS column $(30 \mathrm{~m} \times 0.25 \mathrm{~mm} \times 0.25 \mu \mathrm{m}$; Agilent
Technologies, Inc.) and analyzed on an Agilent GC 7890A coupled with a single quadrupole MSD 5975C (Agilent Technologies) system. For the GC-MS analysis, $1 \mu \mathrm{L}$ of the samples was injected (splitless) with the GC oven kept at $60{ }^{\circ} \mathrm{C}$ for $1 \mathrm{~min}$ after injection, subsequently, the temperature was increased to $325^{\circ} \mathrm{C}$ by $10^{\circ}$ $\mathrm{C} / \mathrm{min}$, followed by a $5 \mathrm{~min}$ hold at $325^{\circ} \mathrm{C}$. Throughout the analysis the injection port temperature was kept constant at $250{ }^{\circ} \mathrm{C}$ for optimal analysis.

\section{GC-MS data processing}

The analysis of the polar metabolites was done by processing the GC-MS raw data files using Metabolite Detector as stated previously [22]. Briefly, the retention indices (RI) calibration was carried out based on the analyses of a mixture of FAMEs internal standards (C8-C28). The data from all GCMS runs were chromatographically aligned after deconvolution and referenced to the FAMEs analyses. In the first instance metabolites were identified by matching experimental spectra to a PNNL augmented version of FiehnLib (PMID: 19928838) library [23], containing spectra and validated retention indices of more than 900 metabolites. Furthermore, unidentified metabolites were screened against the NIST14 GC-MS Spectral Library by comparing spectra alone (denoted with "NIST"). Subsequently, all metabolite identifications were manually validated to reduce deconvolution errors during automated data-processing and to eliminate false identifications. The curated data set of identified metabolites, unidentified features and their abundances for each sample was then further analyzed making use of MetaboAnalyst [24] for the multivariate data analysis (MVDA). Data were median normalized and $\log$ transformed followed by principal component, hierarchical cluster, and heatmap analysis to identify natural clustering within the data.

\section{Results}

Construction and verification of the mutants

A hexokinase/glucokinase double mutant ( $h x k A 1$ glkA4) and a triple mutant (creA $\triangle 4$ hxkA1 glkA4) of Aspergillus nidulans were obtained through sexual crosses (Table 1 and Additional file 3: Figure S1). A reference strain with yellow conidial color (UUAM101.08) was selected from the progeny of the first cross, a hxkA1 glkA4 mutant (UUAM102.37) was selected from the second cross, and a creA $\triangle 4$ (UUAM104.47) as well as the triple mutant creA $\triangle 4$ hxkA1 glkA4 mutant (UUAM104.87) were selected from the third cross for further analysis (as described in the Experimental procedures, Additional file 3: Figure S1).

Phenotypic analysis was performed to visualize the known growth defects and thus confirm the genetic mutations in our selected progeny strains. All strains were grown on D-glucose, D-fructose and D-xylose, using appropriate 
supplements as indicated in the experimental procedures. As expected, growth of the glucokinase/hexokinase double mutant and the triple mutant was impaired on D-glucose and D-fructose (Fig. 1a). The deletion of creA affected growth on all carbon sources tested (Fig. 1a and b). Moreover, its growth was reduced on starch and starch plus Dglucose compared to the reference, although a halo was observed around the colony which was smaller in the reference (Fig. 1b). Halo formation on starch plates around the colony is indicative of the activity of starch-degrading enzymes, converting non-soluble starch into soluble oligosaccharides, effectively 'clearing' the media. In the presence of 3\% D-glucose or D-xylose, CreA represses the production of these enzymes, resulting in the absence of a halo. However, when $c r e A$ is deleted, the halo is observed even in the presence of 3\% D-glucose or D-xylose. The double and triple mutant failed to grow on starch plus $\mathrm{D}$-glucose but not on starch plus D-xylose where a halo was also observed around the colony (Fig. 1b). These results confirmed the expected phenotypes of the selected strains.

\section{Growth profiling on plant biomass substrates revealed different phenotypes between the reference and the mutant strains}

Growth of the reference strain and mutants was further analyzed on 12 pure polysaccharides and untreated agricultural waste substrates to determine the importance of glycolysis for growth (Fig. 2).

On all the selected carbon sources (except cottonseed pulp) the creA $\triangle 4$ mutant grew more strongly than the $h x k A 1$ glkA4 and creA $\triangle 4$ hxkA1 glkA4 strains (Fig. 2). The hexokinase/glucokinase negative mutant was unable to grow on guar gum and sugar beet pulp, whereas growth was strongly reduced on the other tested carbon sources. On xylan, citrus pulp, guar gum, soybean hulls and in particular wheat bran and sugar beet pulp, the carbon catabolite derepressed triple mutant grew better than the hexokinase/glucokinase double mutant (Fig. 2). Thus, growth of the double mutant on these agricultural waste substrates can be partially restored by removing carbon catabolite repression. Wheat bran is an agriculture based crude substrate and a by-product of the wheat milling industry. It consists mainly of (glucurono) arabinoxylan, cellulose, and starch, so is a good source of fermentable sugars such as D-glucose, D-xylose, and L-arabinose. Several substrates demonstrated a strong difference in growth between the double and triple mutant, but we selected wheat bran for further analysis in this study as this substrate has already been studies extensively in other studies in our lab. The composition of the polymeric and crude substrates was analyzed (Additional file 4: Table S1), but this did not reveal any obvious correlation between phenotype and the composition of the substrates. On arabinoxylan and cotton seed pulp there was no difference between the mutants. On apple pectin, creA $\Delta 4$ showed improved growth compared to the triple mutant and the double mutant. The difference between creA $\triangle 4$ hxkA1 glkA4 and $h x k A 1$ glkA4 was less visible than on wheat bran. By comparing the composition of arabinoxylan, cotton seed pulp and apple pectin to the other carbon sources tested where the differences in phenotypes were more pronounced, there was no obvious correlation between phenotype and sugar composition. This suggests that the phenotypic differences between these substrates are likely due to other factors, such as trace elements or inhibiting compounds present in the substrates.

\section{Sugars do not accumulate in cultures of $A$. nidulans reference and mutant strains grown on wheat bran}

Accumulation of sugars in the extracellular medium after transfer of the reference and mutants on wheat bran was analyzed based on the number of reducing groups that could be detected. The results showed that a

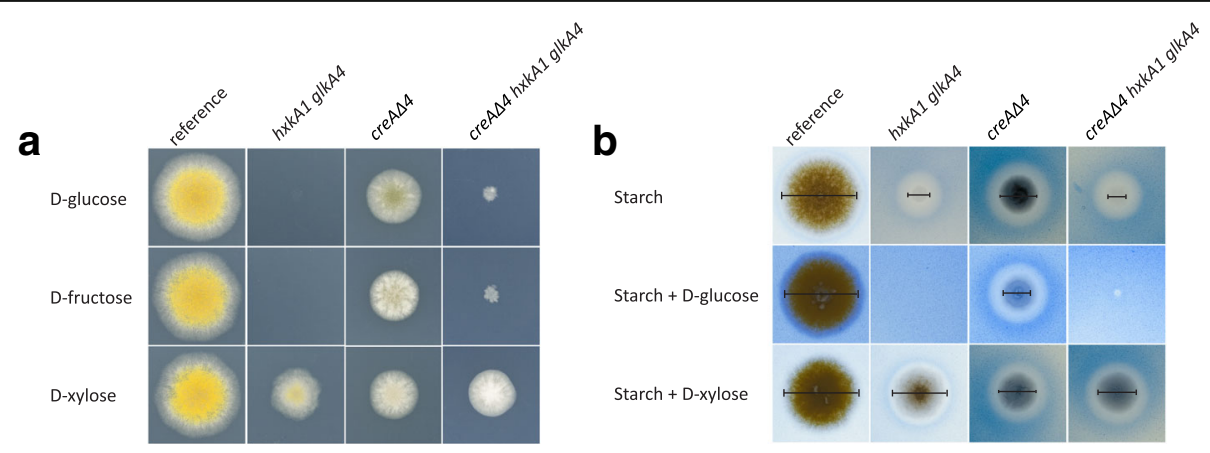

Fig. 1 Phenotype verification of $h \times k A 1 / g / k A 4, c r e A \Delta 4$ and $c r e A \Delta 4 / h x k A 1 / g / k A 4$. a Growth of the reference and the mutants on glucose, fructose and xylose. The A. nidulans strains were grown on MM with $25 \mathrm{mM} \mathrm{D}$-glucose, $25 \mathrm{mM}$ D-fructose and $25 \mathrm{mM} \mathrm{D-xylose} \mathrm{for} 2.5$ days at $37{ }^{\circ} \mathrm{C}$. $\mathbf{b}$ Growth of the reference and the mutants on starch. The A. nidulans strains were grown on MM with $1 \%$ starch with $1 \%$ starch azure, on $1 \%$ starch plus $3 \%$ D-glucose with $1 \%$ starch azure plus $3 \%$ D-glucose and on $1 \%$ starch plus 3\% D-xylose) with $1 \%$ starch azure plus $3 \%$ D-xylose for 3 days at $37^{\circ} \mathrm{C}$. Spore inoculations were done with $5 \times 10^{5}$ spores $/ \mathrm{ml}$. The bars represent the diameter of the colonies 


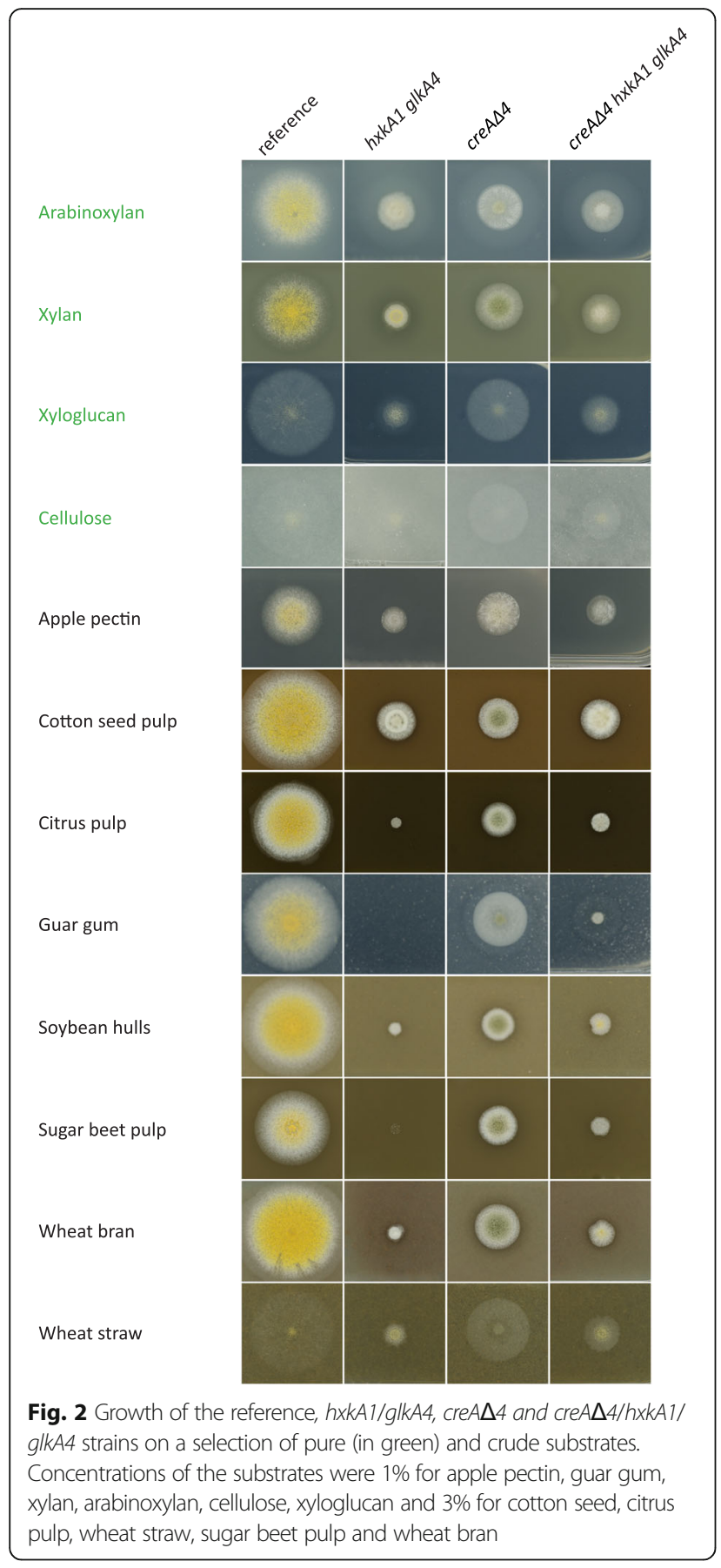

high extracellular sugar level was present for the triple mutant after $8 \mathrm{~h}$ of cultivation, whereas no sugars were detected for the reference and the creA $\Delta 4$ strain. Sugars were also present in the extracellular medium of the double mutant after $8 \mathrm{~h}$ of transfer to wheat bran, but at five-fold lower concentration compared to the triple mutant (Fig. 3a). After $24 \mathrm{~h}$ of transfer to wheat bran, the sugar concentration in the triple mutant is reduced to a very low level (Fig. 3a).
To determine any changes in sugar composition, all strains were pre-grown in liquid cultures containing $\mathrm{MM}$ and the necessary vitamins with xylose (since the $h x k A / g l k A$ mutant does not grow on glucose or fructose) and then transferred to the same medium with wheat bran. After 2, 8 and $24 \mathrm{~h}$ the monosaccharide content in the media was analyzed (Fig. 3b-d).

Significant differences in glucose concentration were found between the triple mutant and $c r e A \Delta 4$ strain. After $8 \mathrm{~h}$ of transfer, a high level of glucose $(31.1 \pm 7.4 \mathrm{mM})$ was detected in the triple mutant, while in the creA $\Delta 4$ strain glucose was not detectable (Fig. 3b). The concentration of glucose were similar in the reference and double mutant at all time points, but five-fold lower compared to the triple mutant after $8 \mathrm{~h}$. In the cre $A \Delta 4$ strain, glucose was only detected after $2 \mathrm{~h}$ of transfer to wheat bran.

Xylose was not detected in cultures of the reference strain and the double mutant (Fig. 3c). However, after $2 \mathrm{~h}$ of transfer to wheat bran, a high level of xylose was detected in the creA $\Delta 4$ (from 4 to $8 \mathrm{mM}$ ) and triple mutant $(6.5 \mathrm{mM})$, which reduced in both mutants after $8 \mathrm{~h}$ (Fig. 3c). This trend was also observed in the creA $\Delta 4$ and triple mutant for arabinose, but at a very low level (Fig. 3d). Low levels of arabinose were also detected in the reference and double mutant at all the time points tested (Fig. 3d). After $24 \mathrm{~h}$ of transfer to wheat bran no glucose, xylose or arabinose was detected in the media of any of the strains tested (Fig. 3b-d).

Thus, our analysis revealed that sugars are not progressively accumulating extracellularly but are consumed during growth of both the double and triple mutant on wheat bran. This was expected for D-xylose and L-arabinose, but the absence of free glucose after $24 \mathrm{~h}$ suggests alternative conversion of glucose.

To trace the conversion products of glucose, GC-MS metabolomic analysis was performed on intracellular metabolite samples from the mycelia of $A$. nidulans at $2 \mathrm{~h}$, $8 \mathrm{~h}$ and $24 \mathrm{~h}$ after transfer to the $1 \%$ wheat bran media. 134 metabolites were detected (Additional file 5: Table S2) , of which 79 could be identified by matching them to entries in the Agilent Fiehn Metabolomics RTL and NIST GC-MS libraries. Metabolite analysis in the creA $\Delta 4$ and triple mutants showed intracellular accumulation of glucose after $2 \mathrm{~h}$. After $8 \mathrm{~h}$ the level is lower and after $24 \mathrm{~h}$ no glucose was detected (Fig. 4). For fructose, we also observed an intracellular accumulation in the creA $A 4$ and triple mutant after $2 \mathrm{~h}$ of transfer to wheat bran. After $8 \mathrm{~h}$, the accumulation was only observed in the creA $\triangle 4 \mathrm{mu}-$ tant, and at $24 \mathrm{~h}$ no fructose was detected. Identified metabolite profiles related to sugar catabolism in $A$. nidulans were similar for the $h x k A 1$ glkA4 mutant compared to the reference and for creA $\Delta 4$ compared to the triple mutant. After $24 \mathrm{~h}$ of transfer to wheat bran medium, no glucose or fructose was detected in any of the strains (Additional file 5: 

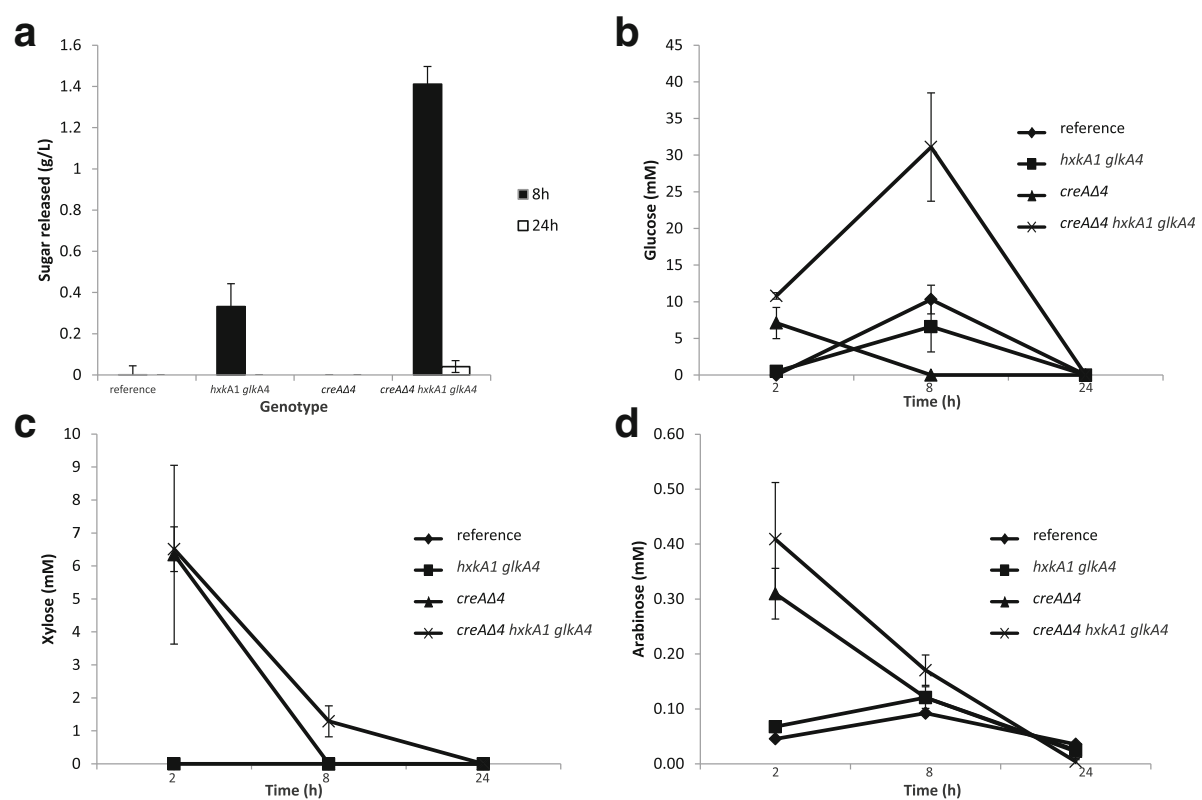

Fig. 3 Sugar consumption by Aspergillus nidulans reference and mutants. All A. nidulans strains were transferred to 1\% wheat bran. a Sugar levels in the extracellular medium of the reference, $h \times k A 1 / g / k A 4, c r e A \Delta 4$ and creA $4 / / h \times k A 1 / g / k A 4$ after $8 \mathrm{~h}$ and $24 \mathrm{~h}$ of transfer to wheat bran. The asterisk indicates a significant difference for glucose after $8 \mathrm{~h}$. b HPAEC-PAD monosaccharide analysis of glucose, (c) xylose and (d) arabinose from A. nidulans reference (dark rhombus), hxkA1/glkA4 (dark square), creA 44 (dark triangle) and creA $4 / \mathrm{hxkA1/g} k \mathrm{kA} 4$ (dark cross) after $2 \mathrm{~h}, 8 \mathrm{~h}$ and $24 \mathrm{~h}$ of transfer to wheat bran. A significant difference was identified between the triple mutant and creA $\Delta 4$ for glucose after $8 \mathrm{~h}(p>0.05$ in students t-test). Means and SD (errors bars) were calculated from three technical replicates

Table S2). In summary, the hexokinase/glucokinase deficient strains when grown on a complex substrate like wheat bran did not show any indications of accumulation of glycolytic intermediates after $24 \mathrm{~h}$ of growth.

\section{Most of the up-regulated genes in the triple mutant compared to the $c r e A \Delta 4$ strain are assigned to the metabolism class}

RNA-sequencing was carried out using RNA isolated from xylose pre-grown mycelium from all four strains that were then transferred to MM with the necessary supplements and $1 \%$ wheat bran, to examine changes in gene expression profiles as a result of the hexokinase glucokinase defect in two genetic backgrounds (reference and creA $\Delta 4$ ). Thus in the next paragraphs, we compared the three mutants to the reference strain, and the same for the cre $A \Delta 4$ background.

To classify the function of the predicted $A$. nidulans genes, the functional catalogue (FunCat) was used [19]. Out of the total 10,460 genes predicted in the genome of A. nidulans, 5812 were associated with FunCat (component, function, and process) that were assigned to 19 main groups (see legend Additional file 6: Figure S2). There were 1798 genes up-regulated in the triple mutant compared to the creA $\Delta 4$ strain, while 814 were up-regulated in the double mutant compared to the reference. Considering all up-regulated genes together, Metabolism was the major class with $39 \%$, followed by Unclassified Proteins
(34\%), Transcription (5.5\%) and Cellular Transport and Transport Mechanisms (5\%).

Analysis of the genes from the Metabolism class was made in these two comparisons. There were 8 subgroups found in this FunCat class (see legend Additional file 6: Figure S2). C-compound and carbohydrate metabolism was the largest subgroup in the Metabolism class with $43 \%$ (307 genes) in the triple mutant vs the cre $A \Delta 4$ strain of the total of up-regulated genes (Additional file 6: Figure $\mathrm{S} 2$ ). In the double mutant, $50 \%$ of the up-regulated genes in the Metabolism class belong to the C-compound and carbohydrate metabolism subgroup (170 genes). Gene function of all genes assigned to the C-compound and carbohydrate metabolism subgroup was analyzed (Additional file 7: Table S3). Among the up-regulated genes assigned to the $\mathrm{C}-\mathrm{CM}$ group, the $s w o M$ gene encoding the glucose-6-phosphate isomerase, two genes involved in the PCP (xylitol dehydrogenase: $x d h A$ and Larabitol dehydrogenase: $\operatorname{lad} A$ ) and four genes involved in the PPP (D-ribulokinase: $r b t A$; D-ribulose-phosphate-3 epimerase: rpeA; glucose-6-phosphate-1-dehydrogenase: $g s d A$ and transaldolase: $p p p A$ ) were up-regulated in the triple mutant compared to creA $\Delta 4$ (Additional file 7: Table S3, sheet 1). In the double mutant (Additional file 7: Table S3, sheet 2), an additional gene involved in the PPP (6phosphogluconate dehydrogenase: $g n d A$ ) was up-regulated compared to the reference. 


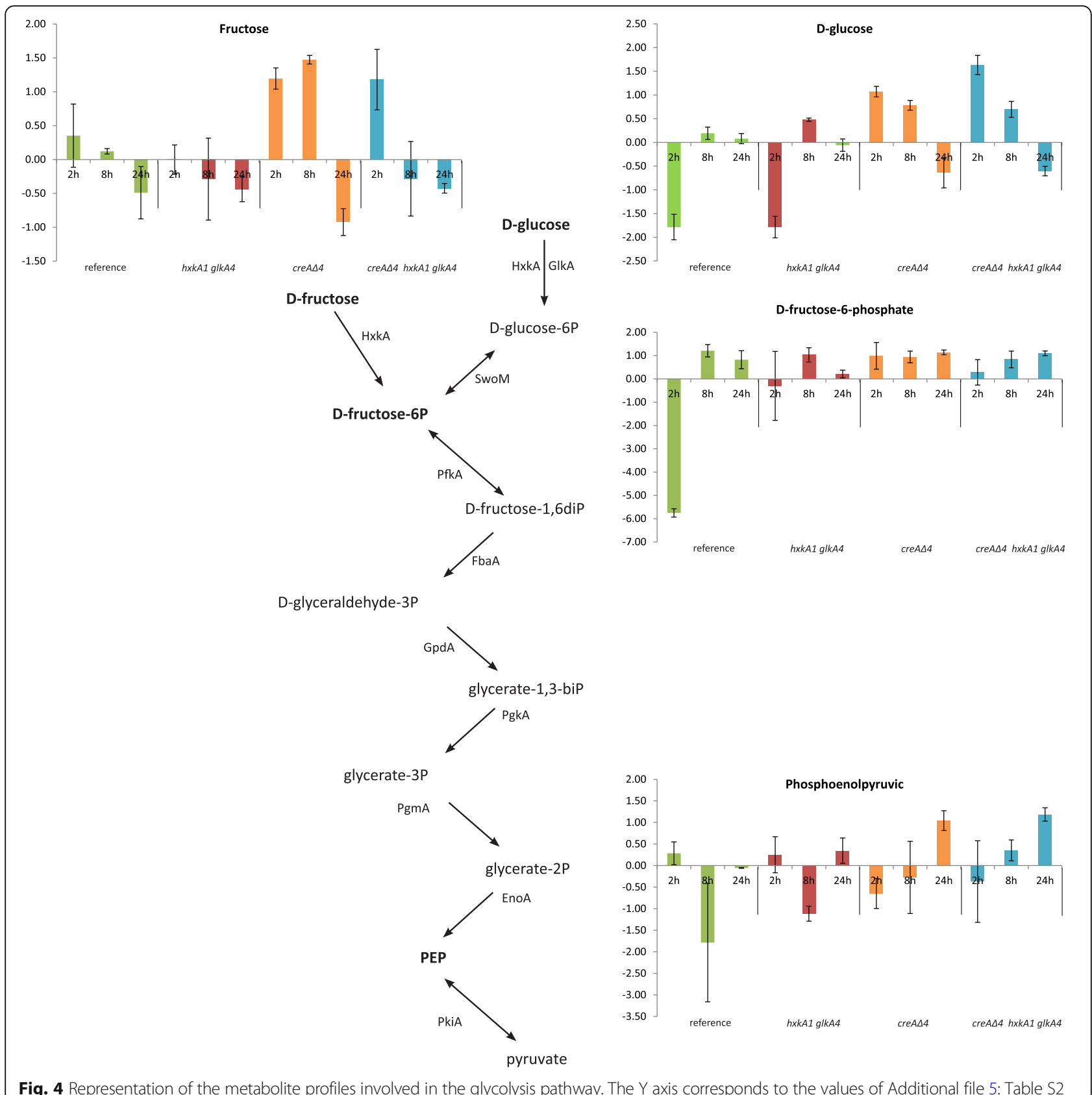

The reduced ability to grow on wheat bran is not due to a reduced expression of CAZy genes involved in plant biomass degradation

The CAZy genes involved in plant biomass from the Glycoside Hydrolase (GH), Auxilary Activities (AAs), Carbohydrate Esterases (CEs) and Polysaccharide Lyases (PLs) families represented $1.7 \%$ of the total number of genes identified in our RNA-sequencing.

CAZy genes with RPKM values above 150 in any of the four conditions are represented in red in Additional file 8: Table S4 (sheet 2). Most of these highly expressed CAZy genes are involved in xylan and cellulose degradation.
Among the significantly differentially expressed genes (with $>1.5$ fold change and $p$-value $<0.05$ ), there are only 17 down-regulated and 10 up-regulated genes in the $h x k A 1$ glkA4 mutant compared to the reference after a transfer to wheat bran for $2 \mathrm{~h}$ (Additional file 8: Table S4, sheet 1). Interestingly, 6 out of 17 genes involved in starch degradation were down-regulated in the double mutant and these genes include four $\alpha$-glucosidases $(\operatorname{agd} A, \operatorname{agdB}$, $a g d E$ and $a g d G)$, one $\alpha$-amylase $(a m y A)$ and one glucoamylase $(g l a A)$. In addition, two endo-xylanases $(x \ln A$ and $x \ln C)$, two endo-polygalacturonases ( $p g a B$ and $p g x A$ ) and one endo-arabinanase $(a b n C)$ were down-regulated. Three 
genes, two $\alpha$-amylase genes (amyD and amyG) and one $\alpha$ glucosidase gene $(a g d F)$ involved in starch degradation are up-regulated in the $h x k A 1 g l k A 4$ mutant as well as seven other CAZy genes that all encode exo-acting enzymes involved in degradation of xylan, cellulose/xyloglucan, galactomannan and pectin.

Overall the reference and the double mutant showed the same CAZy expression profile, which differed from the two creA background strains. As expected, many CAZy genes are up-regulated at high transcript levels in the creA $\triangle 4$ and the triple mutant (Additional file 8: Table S4, sheet 1). Between these two strains there are 16 genes down-regulated and 33 genes up-regulated. Most of the down-regulated genes are involved in starch (eight genes) and cellulose/xyloglucan (seven genes) degradation. We would like to note that even though expression of these genes was significantly reduced, transcript levels of many genes remain at a high level in the triple mutant. Transcript levels of seven out of 16 genes involved in arabinoxylan degradation were even more increased in the triple mutant, including the endo-xylanase gene $x \ln A$, four putative $\beta$-xylosidases (AN1870, AN7864; $b x l D$, AN8477 and AN2664) and two $\alpha$-arabinofuranosidases (abfB and An7781). In summary, these results indicate that the creA $\triangle 4$ mutation has a stronger effect on CAZy gene expression than the metabolic mutations.

\section{The deletion of creA combined with blocking glycolysis results in an increased expression of pentose catabolic and pentose phosphate pathway genes (PCP and PPP)}

Most glycolytic genes showed a similar profile with a significant difference in expression between the reference and the double mutant and between creA $\Delta 4$ and the triple mutant. As expected, in both mutants in which glycolysis is blocked the expression of 6-phosphofructokinase ( $p f k A$ ) , fructose-biphosphate aldolase $(f b a A)$, glyceraldeyde-3phosphate dehydrogenase $(g p d A)$, phosphoglycerate kinase $(p g k A)$, phosphoglycerate mutase $(\operatorname{pgm} A)$ and enolase $(a c u N)$ were down-regulated (lower than 0.7-fold). Exceptions were glucose-6-phosphate isomerase ( $s w o M)$ and pyruvate kinase $(p k i A)$, which were higher expressed in the hexokinase/glucokinase double mutant in both the reference and the creA $\Delta 4$ background (Fig. 5 and Additional file 2: Table S5).

Two genes from the pentose catabolic pathway (PCP), $x d h A$ and $\operatorname{lad} A$, were up-regulated in the double mutant compared to the reference and in the triple mutant compared to $\operatorname{creA} \Delta 4$ ( $>1.5$ fold, $p$-value $<0.05$ ) (Additional file 2: Table S5). In addition, five pentose phosphate pathway (PPP) genes had a significantly higher expression in the double mutant compared to the reference and in the triple mutant compared to creA $\triangle 4$. These encode transaldolase $(p p p A), \quad$ D-ribulokinase $(r b t A), \quad$ D-ribulose-phosphate-3 epimerase (rpeA), 6-phosphogluconate dehydrogenase
(gndA) and glucose-6-phosphate-1-dehydrogenase ( $g s d A$ ). Wheat bran contains also other monosaccharides such as galactose, mannose and glucuronic acid. However, genes involved in the Leloir pathway, alternative D-galactose pathway, D- mannose pathway and TCA cycle did not show a clear effect of blocking glycolysis at the level of hexose phosphorylation.

No difference in gene expression of other genes that might convert glucose, such as glucose oxidase, glucose dehydrogenase, gluconate dehydratase and several hexokinase-like proteins (HxkB, HxkC, HxkD), was observed in the double and triple mutant compared to their reference strains (Additional file 2: Table S5) [3, 5, 25-27]. In our metabolomic data, we could not find any specific intermediates enabling us to pinpoint the alternative pathway for the conversion of glucose.

\section{Validation of the RNA-seq expression profiles by quantitative RT-PCR (qRT-PCR)}

To validate the expression profiles obtained by RNA-seq analysis, two genes involved in the PCP (xylitol dehydrogenase, L-arabitol dehydrogenase) and one CAZy gene $(\alpha$-arabinofuranosidase; $a b f B)$ were tested by quantitative RT-PCR (Additional file 9: Figure S3). Expression trends of the selected genes obtained by qRT-PCR confirmed those obtained with RNA-seq. The primers used in this experiment are indicated in Additional file 10: Table S6.

\section{Higher $\alpha$-arabinofuranosidase and $\beta$-xylosidase activities were observed in the triple mutant compared to the creA mutant alone}

The same cultures used for RNA-sequencing were also used to measure $\alpha$-arabinofuranosidase, $\beta$-glucosidase, $\beta$ xylosidase and cellobiohydrolase activities in the supernatants of the cultures $8 \mathrm{~h}$ and $24 \mathrm{~h}$ after transfer to wheat bran medium. After $8 \mathrm{~h}$ of transfer, cellobiohydrolase $(\mathrm{CBH})$ and $\beta$-glucosidase (BGL) activities were significantly higher in the reference and the creA $\Delta 4$ mutant compared to the two other mutant strains, respectively (Additional file 11: Figure S4). After $24 \mathrm{~h}$ of transfer to wheat bran, this difference was not observed anymore and cellulolytic activity levels were similar in all strains.

A significant increase in $\beta$-xylosidase (BXL) and $\alpha$ arabinofuranosidase (ABF) activities was observed in the triple mutant in comparison with the $c r e A \Delta 4$ after 8 and 24 h (Additional file 11: Figure S4). No differences in BXL activity were observed between the reference and the double mutant at both time-points.

\section{Discussion}

In this study, our aim was to create an Aspergillus strain that more rapidly releases hexoses from plant biomass but does not metabolize them through glycolysis, so they can be fermented by yeast more efficiently to produce bio-fuels. 


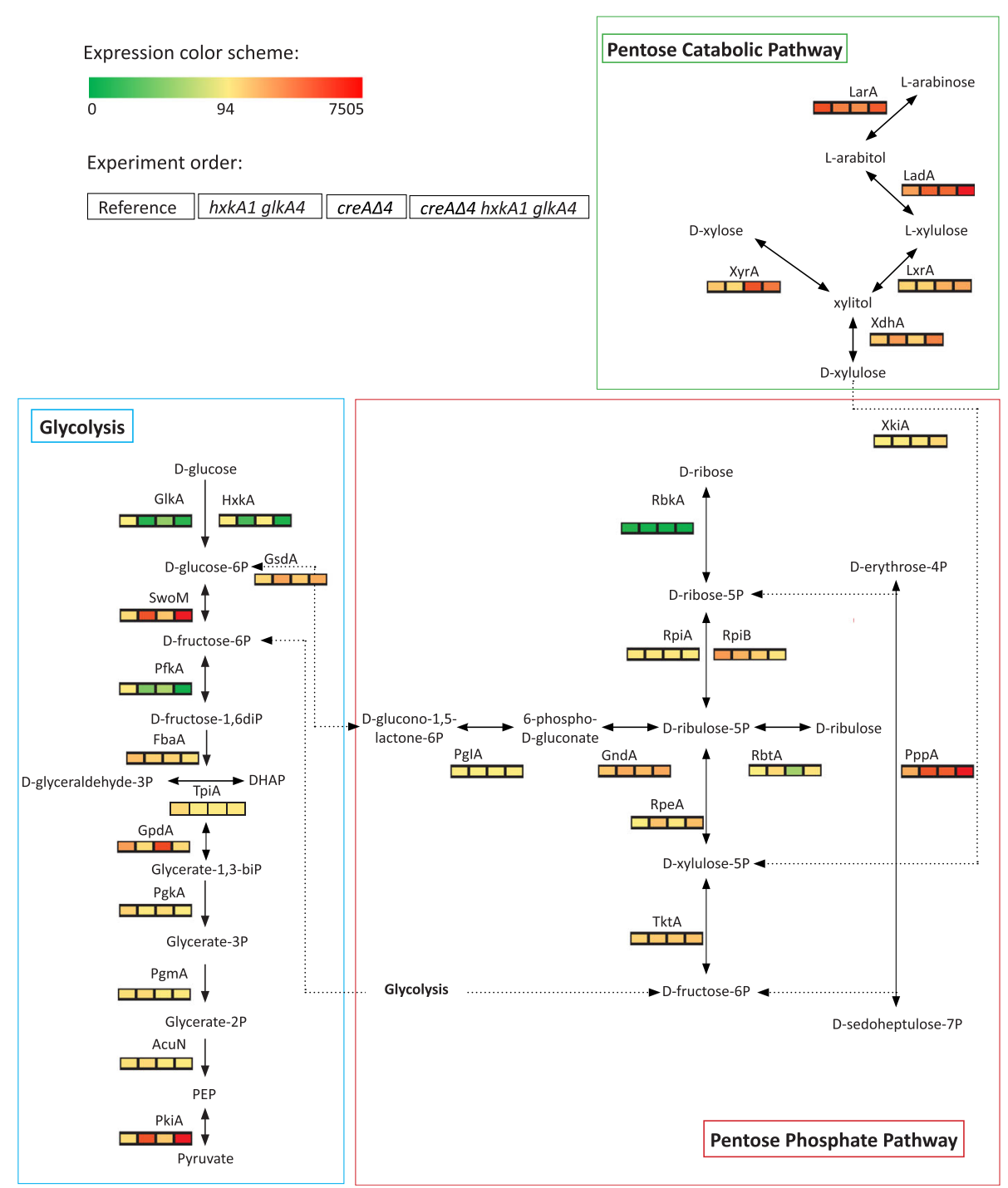

Fig. 5 Schematic representation of the expression of genes of glycolysis, pentose phosphate pathway (PPP) and pentose catabolic pathway (PCP) in Aspergillus nidulans after $2 \mathrm{~h}$ of transfer to $1 \%(\mathrm{~W} / \mathrm{V})$ wheat bran. Gene expression values are presented under the genes and indicated by a color gradient. A decrease in expression is indicated by green squares and increased expression is indicated by a red square. Gene expressions are average values of two biological replicates. The units used is fragments per kilobase of exon per million reads mapped (FPKM). Genes involved in the glycolysis: glucokinase (glkA; AN8689), hexokinase (hxkA; AN7459), glucose-6-phosphate isomerase (swoM; AN6037), phosphofructokinase (pfkA; AN3223), fructosebiphosphate aldolase (fbaA; AN2875), dihydroxyacetone phosphate (DHAP), triose-phosphate isomerase (tpiA; AN6900), glyceraldeyde-3-phosphate dehydrogenase (gpdA; AN8041), phosphoglycerate kinase (pgkA; AN1246), phosphoglycerate mutase (pgmA; AN3059), enolase (acuN; AN5746) and pyruvate kinase (pkiA; AN5210). Genes involved in the PCP: L- arabinose reductase (larA; AN7193), L-arabitol dehydrogenase (IadA; AN0942), L-xylo-3hexulose reductase (IxrA; AN10169), D-xylose reductase (xyrA; AN0423), xylitol dehydrogenase ( $x d h A ;$ AN9064) and D-xylulose kinase (xkiA; AN8790). Genes involved in the PPP: glucose-6-phosphate-1-dehydrogenase (gsdA; AN2981), 6-phosphogluconolactonase (pglA; AN0285), 6-phosphogluconate dehydrogenase (gndA; AN3954), D-ribulose-phosphate-3 epimerase (rpeA; AN7588), transketolase (tktA; AN0688), D-ribulokinase (rbtA; AN6985), transaldolase (pppA; AN0240), ribose 5-phosphate isomerase (rpiA; AN2440), ribose 5-phosphate isomerase (rpiB; AN5907) and ribokinase (rbkA; AN7995)

As a first step to achieve this, mutants for hexokinase, glucokinase, creA and combinations thereof were constructed in $A$. nidulans and the resulting strains were evaluated for their ability to accumulate hexoses.

HxkA and GlkA represent the only active hexose kinases of $A$. nidulans, even though other genes encoding putative hexose phosphorylating activities, such as the putative $\mathrm{HxkB}$, are present in the genome of $A$. nidulans. However, a previous study in Aspergillus fumigatus reported that recombinant HxkB has no sugar-phosphorylating activity [8].

The phenotype of the $h x k A 1$ glkA4 strains [5] was confirmed and the double mutant was unable to grow on glucose and fructose. The creA $\Delta 4$ mutant has been described as a strongly derepressed creA mutant [28] and was shown to have elevated polysaccharide degrading enzyme activities [29]. Our RNA-seq data 
indicate that this is a transcriptionally down-regulated creA allele.

First, we evaluated the changes in the extracellular sugars in the reference and in all the mutant strains. A 3,5-dinitrosalicylic acid (DNS) sugar assay showed that free sugars were only present in the culture supernatant at the early time point in the triple mutant, while after $24 \mathrm{~h}$ of incubation, sugar levels were close to zero in all the strains. This suggests that the balance of sugar release and sugar uptake had reached a stable state. This is in agreement with the results obtained from the HPAEC analysis. At $8 \mathrm{~h}$, glucose was higher in the triple mutant compared to the creA $\triangle 4$, but after $24 \mathrm{~h}$ no glucose was detected for any of the strains. Interestingly, fructose and other monosaccharides did not accumulate in all strains after $2 \mathrm{~h}, 8 \mathrm{~h}$ and $24 \mathrm{~h}$ of transfer to wheat bran. This indicates that hexoses are still being consumed in the hxkA1 glkA4 negative strains suggesting that alternative pathways are converting them.

In the hxkA1 glkA4 mutant, a set of genes encoding starch degrading enzymes was down-regulated at $2 \mathrm{~h}$ on wheat bran compared to the reference. In the triple mutant, expression of both starch and cellulose genes were reduced compared to the $\mathrm{cre} A \Delta 4$ strain. Most glycolytic genes were down-regulated in both double and triple mutant as well suggesting blocking glycolysis caused an initial negative feedback of extracellular D-glucose release from starch and cellulose. The $\beta$-glucosidase (BGL) and cellobiohydrolase $(\mathrm{CBH})$ activities also decreased after $8 \mathrm{~h}$ of transfer to wheat bran in both hxkA1 glkA4 negative strains. After $24 \mathrm{~h}$ of incubation, BGL and CBH enzyme activities were at a similar level as their reference strain. Together with the extracellular sugar analysis, this again suggests that during growth on wheat bran over time a reorganization of central metabolism occurs in the $h x k A 1$ glkA4 negative strains to regulate $\mathrm{D}$-glucose release and re-direct glucose conversion.

Growth profiles of the $h x k A 1 / g l k A 4$ negative strains on plant biomass and pure polysaccharides were performed to determine the importance of glycolysis for growth on these substrates. Previously it was shown that the Aspergillus fumigatus hexokinase/glucokinase double deletion mutant failed to grow on glucose, fructose, mannose, sorbose, glucosamine, and saccharose [8]. Our results showed that in all the carbon sources tested (except arabinoxylan, xyloglucan, and cotton seed pulp) the double mutant had reduced growth compared to the triple mutant. The improved growth of our triple mutant in comparison with the double mutant observed for most substrates is most likely a consequence of the increased transcript levels of CAZymes due to the creA $\Delta 4$ mutation.

Our extracellular sugar analysis suggests that glucose and fructose may be converted through an alternative metabolic pathway. RNA-sequencing analysis allowed us to analyze in more detail the effect of the metabolic mutations and to possibly identify the pathway through which glucose and fructose were converted. Since in this biomass the amounts of galactose and mannose are low, these pathways are not expected to substantially contribute to growth. Therefore, the PCP and PPP should be the most relevant pathways necessary for growth. We assumed that the metabolic defects introduced in the strains of this study do not affect sugar transport.

Most of the glycolytic genes were down-regulated in the $h x k A 1$ glkA4 strains in both the reference and creA $\triangle 4$ background. However, glucose-6-phosphate isomerase, encoded by $s w o M$, which converts glucose-6-phosphate to fructose-6-phosphate in a reversible reaction, was significantly up-regulated in the strains not able to phosphorylate glucose. The pentose phosphate pathway generates besides NADPH both fructose-6-phosphate and glyceraldehyde-3-phosphate. The strong up-regulation of swoM indicates the necessity to increase the flux through the PPP by generating glucose-6-phosphate from the available fructose-6-phosphate pool. This is also consistent with the up-regulation of the expression of $g s d A$ and gndA, involved in of two of three steps of the oxidative PPP, which is more evident in the hexokinase/glucokinase negative background strains. The pentoses xylose and arabinose are the main carbon sources available for catabolism and these pathways require NADPH [30]. Xylose and arabinose represent $34 \%$ and $16 \%(w / w)$, respectively of the wheat bran. The $x d h A$ gene, encoding xylitol dehydrogenase that converts xylitol into D-xylulose, and ladA, encoding L-arabitol dehydrogenase that converts Larabitol into L-xylulose, were significantly up-regulated in the $h x k A 1 / g l k A 4$ negative strains. Also $x k i A$, encoding Dxylulose kinase that converts D-xylulose into D-xylulose5-phosphate, was higher in the triple mutant. An important response in the non-oxidative PPP is also observed in the $h x k A 1 / g l k A 4$ negative backgrounds which showed an increased expression of rpeA encoding the epimerase responsible for converting $\mathrm{D}$-xylulose-5-phosphate into D-ribulose-5-phosphate and the pppA gene encoding a transaldolase. The ribose-5-phosphate isomerase encoding gene rpiA hardly changes its response in the mutant backgrounds whereas rpiB expression is the highest in the reference strain. Transcriptional regulation seems not to be necessary at this level. Subsequently, in the final steps of the pathway fructose-6-phosphate and glyceraldehyde3-phosphate are generated. Glyceraldehyde-3-phosphate is then available for the final part of the glycolytic pathway which can then enter the TCA cycle.

The pool of fructose-6-phosphate generated by the catabolism of the pentoses will, in accordance with the up-regulation observed in the oxidative part of the PPP, be partially recycled whereas it will also be used for biosynthetic purposes by converting fructose-6-phosphate 
to glucose-1-phosphate via a glucose-6-phosphate isomerase (SwoM) and a phosphoglucomutase (PgmB). The pgmB gene and those encoding the subsequent steps are not up-regulated in the mutants. However, a putative UTP-glucose-1-phosphate uridylyltransferase encoding gene, galF, responsible for conversion of glucose-1phosphate into UDP-glucose, was up-regulated in the triple mutant. Also, a gene encoding a putative glycogen synthase, which converts UDP-glucose into glycogen, was up-regulated in the double and triple mutant. An excess of the fructose-6-phosphate pool may lead to fructose-6-phosphate phosphatase activity. Interestingly, a fructose-2,6-bisphosphate 2-phosphatase encoding gene $(f b p Z)$ was up-regulated in the triple mutant compared to the $c r e A \Delta 4$ strain. This is likely to lead to lower levels of fructose-2,6-biphosphate and as a consequence to a diminished activation of the fructose-6-phosphate kinase [31]. Fructose observed intracellularly at early time points in the creA deletion strain and the triple mutant may arise by fructose-6-phosphate phosphatase activity which could be the consequence of an accumulation of the fructose-6-phosphate pool. However, the presence of a glucose isomerase that could convert Dglucose into D-fructose cannot be excluded. Interestingly, $\operatorname{dak} A$ (encoding dihydroxyacetone kinase) was upregulated in strains in which $h x k A$ and $g l k A$ were mutated, especially in the creAd4 background. This could indicate that this enzyme is also involved in another pathway, such as fructose-6-phosphate accumulation via xylulose-5P and DHA.

These results could explain the strong up-regulation observed in the hxkA1/glkA4 strains in both the reference and creA $\triangle 4$ background of $s d h A$, encoding sorbitol dehydrogenase that converts fructose into sorbitol. Therefore, the conversion of fructose-6-phosphate may occur via a phosphatase and subsequently SdhA. Another alternative for the fructose-6-phosphate conversion might be through mannitol-1-phosphate 5-dehydrogenase, encoded by $m d p A$, which converts fructose-6-phosphate into mannitol-1-phosphate. The $m d p A$ gene is highly expressed in all strains. The accumulation of mannitol in A. nidulans when cultivated on glucose or fructose due to mannitol-1-phosphatase activity is well known [32-35]. Extracellular metabolite analysis showed high levels of mannitol in the creA $\triangle 4$ and in the triple mutant after $2 \mathrm{~h}$ of transfer to wheat bran (data not shown).

In summary our hypothesis is that xylose and arabinose are converted into fructose-6-phosphate and glyceraldehyde-3-phosphate. Fructose-6-phosphate will be converted into glucose-6-phosphate through the PPP, into mannitol through MpdA and mannitol-1phosphatase as well as sorbitol through a phosphatase and subsequently SdhA. Expression data suggests the latter pathway to prevail.
The RNA-seq results were also used to evaluate the expression of other metabolic genes that might convert glucose. Glucose oxidase activity would result in the formation of D-gluconate and hydrogen peroxide. Our RNAseq data did not show a significant up-regulation in the double mutant compared to the reference strain and in the triple mutant compared to the creA mutant. Also, in the metabolomics analysis we did not detect gluconate. Since glucose seems not to be oxidized, another option would then be reduction to glucitol. In the metabolomic analysis we also did not detect glucitol. Glucose could also be converted into trehalose, since we observed formation of glucose-6-phosphate from the PPP whereas also glucose-1-phosphate and UDP-glucose biosynthesis is intact. But most likely, glucose is either directly reduced or converted to fructose followed by production of polyols.

\section{Conclusion}

In conclusion, this study provides an in-depth analysis of the effect of a hexokinase/glucokinase double mutant at the gene expression level during growth of $A$. nidulans on an agricultural waste product. Although glucose and fructose initially accumulate, these hexoses are converted through alternative metabolic pathways. A more detailed metabolomic analysis is required to determine which pathways are actually used and what the final products are. The deletion of $\mathrm{creA}$ in combination with a hexokinase glucokinase deficiency results in an increased expression of the PCP and PPP. This indicates that the reduced ability to use hexoses as carbon source has resulted in a shift towards the pentose fraction of wheat bran as the major carbon source to support growth.

\section{Additional files}

Additional file 1: Figure S5. Principal component analysis (PDF $165 \mathrm{~kb}$ ) Additional file 2: Table S5. Expression of known gene involved in central carbon metabolism in Aspergillus nidulans. (XLSX 32 kb)

Additional file 3: Figure S1. Scheme of sexual crossing in Aspergillus nidulans. (PDF $185 \mathrm{~kb}$ )

Additional file 4: Table S1. Composition of the substrates. (XLSX $13 \mathrm{~kb}$ )

Additional file 5: Table S2. Intracellular metabolism of Aspergillus nidulans during growth in wheat bran. (XLSX 84 kb)

Additional file 6: Figure S2. Functional classification of Aspergillus nidulans genes according to FunCat. (PDF $151 \mathrm{~kb}$ )

Additional file 7: Table S3. Functional classification of A. nidulans genes belonging to the C-compound and carbohydrate metabolism subclass according to FunCat. (XLSX $60 \mathrm{~kb}$ )

Additional file 8: Table S4. Expression of selected CAZymes involved in the degradation of plant biomass in Aspergillus nidulans. (XLSX 63 kb)

Additional file 9: Figure S3. Expression patterns and validation of RNA-sequencing analysis by qPCR. (PDF $325 \mathrm{~kb}$ )

Additional file 10: Table S6. Primers used in this study to generate the gene fragments for qRT-PCR analysis. (XLSX $10 \mathrm{~kb}$ )

Additional file 11: Figure S4. Comparison of extracellular enzyme activities in reference and disruption strains. (PDF $169 \mathrm{~kb}$ ) 


\section{Abbreviations}

AA: Auxiliary activities; CAZy: Carbohydrate active enzyme database; CCR: Carbon catabolite repression; CE: Carbohydrate esterase; creA: Carabon catabolite repressor encoding gene; $\mathrm{GH}$ : Glycoside hydrolase; glkA: Glucokinase encoding gene; hxkA: Hexokinase encoding gene; PCP: Pentose catabolic pathway; PL: Polysaccharide lyase; PPP: Pentose phosphate pathway

\section{Acknowledgements}

We thank Adiphol Dilokpimol for his help with the HPAEC- PAD analysis and Sylvia Klaubauf for her help regarding the statistical analysis.

\section{Funding}

CK, EB and MVA-P were supported by a grant of the Dutch Technology Foundation STW, Applied Science division of NWO, and the Technology Program of the Ministry of Economic Affairs 016.130.609 to RPdV. SEB, HH and Y-MK were supported by the U.S. Department of Energy (DOE), Office of Science, Office of Biological and Environmental Research (OBER) Genomic Science program, under Award Number DE- SC0008744. Part of this research was performed at the Environmental Molecular Sciences Laboratory (EMSL), a national scientific user facility sponsored by the U.S. DOE OBER, and located at PNNL. PNNL is a multiprogram national laboratory operated by Battelle for the DOE under Contract DE-AC05-76RLO 1830.

The funding bodies did not participate in the design of the study nor in collection, analysis, and interpretation of data and in writing the manuscript, but only provided us with the funds to do so.

\section{Availability of data and materials}

The data sets supporting the results of this article are available in the GEO repository with accession number: GSE94775.

\section{Authors' contributions}

RPdV conceived the study. RPdV and EB designed experiments. SD, CK and RSK performed the experiments. MVAP and MZ performed the bioinformatics analysis. $\mathrm{HMH}, \mathrm{YMK}$ and SEB performed the metabolomics analysis. CK analysed the data. $C K, E B$, JV and RPdV interpreted the data and wrote the manuscript. All authors read and approved the final manuscript.

\section{Ethics approval and consent to participate}

Not applicable.

\section{Consent for publication}

Not applicable.

\section{Competing interests}

The authors declare that they have no competing interests.

\section{Publisher's Note}

Springer Nature remains neutral with regard to jurisdictional claims in published maps and institutional affiliations.

\section{Author details}

${ }^{1}$ Fungal Physiology, Westerdijk Fungal Biodiversity Institute \& Fungal Molecular Physiology, Utrecht University, Uppsalalaan 8, 3584, CT, Utrecht The Netherlands. ${ }^{2}$ Microbiology, Utrecht University, Padualaan 8, 3584, CH, Utrecht, The Netherlands. ${ }^{3}$ Earth and Biological Sciences Directorate, Pacific Northwest National Laboratory, Richland, WA, USA. ${ }^{4}$ Fungal Genetics and Technology Consultancy, P.O. Box 396, 6700, AJ, Wageningen, The Netherlands.

\section{Received: 25 October 2017 Accepted: 19 March 2018}

Published online: 22 March 2018

\section{References}

1. de Vries RP, Visser J. Aspergillus enzymes involved in degradation of plant cell wall polysaccharides. Microbiol Mol Biol Rev. 2001;65(4):497-522.

2. Panneman H, Ruijter GJ, van den Broeck HC, Driever ET, Visser J. Cloning and biochemical characterisation of an Aspergillus niger glucokinase. Evidence for the presence of separate glucokinase and hexokinase enzymes. Eur J Biochem. 1996;240(3):518-25.
3. Ruijter GJG, Panneman H, van den Broek HC, Bennett JM, Visser J. Characterisation of the Aspergillus nidulans frA1 mutant: hexose phosphorylation and apparent lack of involvement of kexokinase in glucose repression. FEMS Microbiol Lett. 1996;139:223-8.

4. Upadhyay S, Shaw BD. A phosphoglucose isomerase mutant in Aspergillus nidulans is defective in hyphal polarity and conidiation. Fungal Genet Biol. 2006;43(11):739-51.

5. Flipphi M, van de Vondervoort PJ, Ruijter GJ, Visser J, Arst HN Jr, Felenbok B. Onset of carbon catabolite repression in Aspergillus nidulans. Parallel involvement of hexokinase and glucokinase in sugar signaling. J Biol Chem. 2003:278(14):11849-57.

6. Park EY, Naruse K, Kato T. One-pot bioethanol production from cellulose by co-culture of Acremonium cellulolyticus and Saccharomyces cerevisiae. Biotechnol Biofuels. 2012;5(1):64.

7. Tesfaw A, Assefa F. Current trends in bioethanol production by Saccharomyces cerevisiae: substrate, inhibitor reduction, growth variables, coculture, and immobilization. Int Sch Res Notices. 2014:2014:532852.

8. Fleck CB, Brock M. Aspergillus fumigatus catalytic glucokinase and hexokinase: expression analysis and importance for germination, growth, and conidiation. Eukaryot Cell. 2010;9(7):1120-35.

9. Shroff RA, O'Connor SM, Hynes MJ, Lockington RA, Kelly JM. Null alleles of creA, the regulator of carbon catabolite repression in Aspergillus nidulans. Fungal Genet Biol. 1997;22(1):28-38.

10. de Vries R, Burgers K, van de Vondervoort P, Frisvad J, Samson R, Visser J. A new black Aspergillus species, A. vadensis, is a promising host for homologous and heterologous protein production. Appl Environ Microbiol. 2004;70:3954-9.

11. Todd RB, Davis MA, Hynes MJ. Genetic manipulation of Aspergillus nidulans: meiotic progeny for genetic analysis and strain construction. Nat Protoc. 2007:2(4):811-21.

12. Yu S, Olsen CE, Marcussen J. Methods for the assay of 1,5-anhydro-dfructose and a-1,4-glucan lyase. Carbohydr Res. 1997:305(1):73-82.

13. Makela MR, Mansouri S, Wiebenga A, Rytioja J, de Vries RP, Hilden KS. Penicillium subrubescens is a promising alternative for Aspergillus niger in enzymatic plant biomass saccharification. New Biotechnol. 2016;33(6):834-41.

14. Li R, Yu C, Li Y, Lam TW, Yiu SM, Kristiansen K, Wang J. SOAP2: an improved ultrafast tool for short read alignment. Bioinformatics. 2009;25(15):1966-7.

15. Mortazavi A, Williams BA, McCue K, Schaeffer L, Wold B. Mapping and quantifying mammalian transcriptomes by RNA-Seq. Nat Methods. 2008;5:621.

16. Kayala MA, Baldi P. Cyber-T web server: differential analysis of highthroughput data. Nucleic Acids Res. 2012;40(Web Server issue):W553-9.

17. Edgar R, Domrachev M, Lash AE. Gene expression omnibus: NCBI gene expression and hybridization array data repository. Nucleic Acids Res. 2002;30(1):207-10.

18. Cerqueira GC, Arnaud MB, Inglis DO, Skrzypek MS, Binkley G, Simison M, Miyasato SR, Binkley J, Orvis J, Shah P, et al. The aspergillus genome database: multispecies curation and incorporation of RNA-Seq data to improve structural gene annotations. Nucleic Acids Res. 2014;42(D1):D705-D10.

19. Ruepp A, Zollner A, Maier D, Albermann K, Hani J, Mokrejs M, Tetko I, Guldener U, Mannhaupt G, Munsterkotter M, et al. The FunCat, a functional annotation scheme for systematic classification of proteins from whole genomes. Nucleic Acids Res. 2004;32(18):5539-45.

20. Livak KJ, Schmittgen TD. Analysis of relative gene expression data using real-time quantitative PCR and the $2^{-\Delta \Delta C}$ T method. Methods. 2001;25:402-8.

21. Kim YM, Schmidt BJ, Kidwai AS, Jones MB, Deatherage Kaiser BL, Brewer HM, Mitchell HD, Palsson BO, McDermott JE, Heffron F, et al. Salmonella modulates metabolism during growth under conditions that induce expression of virulence genes. Mol BioSyst. 2013;9(6):1522-34.

22. Hiller K, Hangebrauk J, Jager C, Spura J, Schreiber K, Schomburg D. MetaboliteDetector: comprehensive analysis tool for targeted and nontargeted GC/MS based metabolome analysis. Anal Chem. 2009:81(9):3429-39.

23. Kind T, Wohlgemuth G, Lee DY, Lu Y, Palazoglu M, Shahbaz S, Fiehn O. FiehnLib: mass spectral and retention index libraries for metabolomics based on quadrupole and time-of-flight gas chromatography/mass spectrometry. Anal Chem. 2009:81(24):10038-48.

24. Xia J, Sinelnikov IV, Han B, Wishart DS. MetaboAnalyst 3.0—making metabolomics more meaningful. Nucleic Acids Res. 2015:43:W251-7.

25. Katz ME, Masoumi A, Burrows SR, Shirtliff CG, Cheetham BF. The Aspergillus nidulans xprF gene encodes a hexokinase-like protein involved in the regulation of extracellular proteases. Genetics. 2000;156(4):1559-71. 
26. Bernardo SM, Gray KA, Todd RB, Cheetham BF, Katz ME. Characterization of regulatory non-catalytic hexokinases in Aspergillus nidulans. Mol Gen Genomics. 2007;277(5):519-32.

27. Katz ME, Braunberger KS, Kelly JM. Role of HxkC, a mitochondrial hexokinase-like protein, in fungal programmed cell death. Fungal Genet Biol. 2016;97(Supplement C):36-45.

28. Bailey C, Arst HN. Carbon catabolite repression in Aspergillus nidulans. Eur J Biochem. 1975;51(2):573-7.

29. van der Veen P, Arst HN Jr, Flipphi MJ, Visser J. Extracellular arabinases in Aspergillus nidulans: the effect of different cre mutations on enzyme levels. Arch Microbiol. 1994;162(6):433-40.

30. Witteveen CFB, Busink R, Vandevondervoort P, Dijkema C, Swart K, Visser J. L-arabinose and D-xylose catabolism in Aspergillus niger. J Gen Microbiol. 1989;135:2163-71.

31. Mesojednik S, Legisa M. Posttranslational modification of 6-phosphofructo1-kinase in Aspergillus niger. Appl Environ Microbiol. 2005;71(3):1425-32.

32. Witteveen CFB, Visser J. Polyol pools in Aspergillus niger. FEMS Microbiol Lett. 1995;134(1):57-62.

33. Aguilar-Osorio G, vanKuyk PA, Seiboth B, Blom D, Solomon PS, Vinck A, Kindt F, HAB W, de Vries RP. Spatial and developmental differentiation of mannitol dehydrogenase and mannitol-1-phosphate dehydrogenase in Aspergillus niger. Eukaryot Cell. 2010;9(9):1398-402.

34. Diano A, Bekker-Jensen S, Dynesen J, Nielsen J. Polyol synthesis in Aspergillus niger: influence of oxygen availability, carbon and nitrogen sources on the metabolism. Biotechnol Bioeng. 2006;94(5):899-908.

35. Ruijter GJG, Bax M, Patel H, Flitter SJ, van de Vondervoort PJI, de Vries RP, vanKuyk PA, Visser J. Mannitol is required for stress tolerance in Aspergillus niger conidiospores. Eukaryot Cell. 2003;2(4):690-8.

\section{Submit your next manuscript to BioMed Central and we will help you at every step:}

- We accept pre-submission inquiries

- Our selector tool helps you to find the most relevant journal

- We provide round the clock customer support

- Convenient online submission

- Thorough peer review

- Inclusion in PubMed and all major indexing services

- Maximum visibility for your research

Submit your manuscript at www.biomedcentral.com/submit 